\title{
Tumor invasiveness as defined by the newly proposed IASLC/ATS/ ERS classification has prognostic significance for pathologic stage IA lung adenocarcinoma and can be predicted by radiologic parameters
}

\author{
Mamoru Takahashi, MD, ${ }^{\mathrm{a}}$ Yoshiki Shigematsu, MD, PhD, ${ }^{\mathrm{a}}$ Makoto Ohta, MD, ${ }^{\mathrm{b}}$ \\ Hironobu Tokumasu, MD, MPH, ${ }^{\mathrm{c}}$ Tadashi Matsukura, MD, PhD, ${ }^{\mathrm{a}}$ and Takashi Hirai, MD, $\mathrm{PhD}^{\mathrm{a}}$
}

\begin{abstract}
Objectives: The International Association for the Study of Lung Cancer, American Thoracic Society, and European Respiratory Society (IASLC/ATS/ERS) have collaborated to propose a new pathologic classification of lung adenocarcinoma. In this classification, noninvasiveness and invasiveness have been newly defined for lung adenocarcinoma. The aims of this study were to validate the prognostic significance of tumor invasiveness as defined by the new IASLC/ATS/ERS classification and to assess the relationship between pathologic invasiveness and radiologic findings in pathologic stage IA lung adenocarcinoma.
\end{abstract}

\begin{abstract}
Methods: We retrospectively reviewed 123 consecutive patients with pathologic stage IA lung adenocarcinoma. Pathologic data were classified according to the new IASLC/ATS/ERS classification. The following radiologic parameters were assessed using thin-section computed tomography: the ground-glass opacity ratio, tumor disappearance rate, and consolidation diameter.
\end{abstract}

Results: There were 54 noninvasive and 69 invasive adenocarcinomas. Five-year overall survival rates for noninvasive adenocarcinoma and invasive adenocarcinoma were $100 \%$ and $78.4 \%$, respectively; this difference was statistically significant $(P<.01)$, indicating the prognostic value of this classification. Receiver operating characteristic curves of the ground-glass opacity ratio, tumor disappearance rate, and consolidation diameter identified the optimal cut-off values for predicting the presence of invasive tumors as $50 \%, 75 \%$, and $10 \mathrm{~mm}$, respectively.

Conclusions: We found that by using the new IASLC/ATS/ERS classification, histologic subtypes of pathologic stage IA lung adenocarcinoma with prognostic value could be identified. Tumor invasiveness of lung adenocarcinoma as defined by this classification can be predicted by evaluating the ground-glass opacity ratio, tumor disappearance rate, and consolidation diameter on thin-section computed tomography. (J Thorac Cardiovasc Surg 2014;147:54-9)

The 2004 World Health Organization classification and the Noguchi classification are the most widely used systems for lung adenocarcinoma. ${ }^{1-3}$ However, rapidly evolving advances in the understanding of lung adenocarcinoma have resulted in confusion about the current classifications. ${ }^{4-6}$ To address this, the International Association for the Study of Lung Cancer, American Thoracic Society, and European Respiratory Society (IASLC/ATS/ERS) proposed a new classification of lung adenocarcinoma in 2011. ${ }^{5}$ Validation of the new IASLC/ ATS/ERS classification will illustrate its effect in the clinical setting. In addition, correlation between radiologic

From the Department of Chest Surgery, ${ }^{\mathrm{a}}$ Department of Pathology, ${ }^{\mathrm{b}}$ Fukui Red Cross Hospital, Fukui, Japan; and Department of Pharmacoepidemiology, ${ }^{\mathrm{c}}$ Graduate School of Medicine and Public Health, Kyoto University, Kyoto, Japan.

Disclosures: Authors have nothing to disclose with regard to commercial support.

Received for publication Feb 8, 2013; revisions received Aug 23, 2013; accepted for publication Aug 29, 2013; available ahead of print Oct 15, 2013.

Address for reprints: Mamoru Takahashi, MD, Fukui Red Cross Hospital, 2-4-1 Tsukimi, Fukui City, Fukui, 918-8501, Japan (E-mail: mt10947@yahoo.co.jp). 0022-5223/\$36.00

Copyright (c) 2014 by The American Association for Thoracic Surgery

http://dx.doi.org/10.1016/j.jtcvs.2013.08.058 findings and tumor invasiveness as defined by the new classification will enable thoracic surgeons to select the optimal surgical procedure. The present study had 2 purposes. First, we validated the prognostic significance of tumor invasiveness as defined by the newly proposed IASLC/ATS/ERS classification for pathologic stage IA lung adenocarcinoma. Second, we clarified the relationship between the radiologic findings on preoperative thin-section computed tomography (CT) images and tumor invasiveness according to the new IASLC/ATS/ ERS classification.

\section{MATERIALS AND METHODS}

Between January 2004 and December 2010, there were 131 patients with preoperatively untreated stage IA adenocarcinoma who underwent lung tumor resection with dissection or sampling of lymph nodes and were evaluated pathologically as having stage IA (p-stage IA) adenocarcinoma at the Fukui Red Cross Hospital.

Of these, we retrospectively evaluated a series of 123 consecutive patients with a single stage IA adenocarcinoma. Eight patients with multiple lung adenocarcinomas were excluded (Figure 1). All surgeries were performed or supervised by the same surgeon (T.H.) throughout the study period. The study included 56 men and 67 women, with a mean age of 67 years (range, 28-85 years). The median follow-up period was 


\section{Abbreviations and Acronyms \\ AIS = adenocarcinoma in situ \\ AUC = area under the curve \\ $\mathrm{BAC}=$ bronchioloalveolar carcinoma \\ $\mathrm{CD}=$ consolidation diameter \\ CEA $=$ carcinoembryonic antigen \\ CT = computed tomography \\ GGO = ground-glass opacity \\ $\mathrm{HU}=$ Hounsfield unit \\ IASLC/ = International Association for the Study ATS/ERS of Lung Cancer, American Thoracic Society, and European Respiratory Society \\ MIA = minimally invasive adenocarcinoma \\ p-stage IA $=$ pathologic stage IA \\ ROC = receiver operating characteristic \\ TDR $=$ tumor disappearance rate}

48 months (range, 19-94 months). Patient consent for review of medical records including preoperative images was obtained. This retrospective study was approved by our institutional review board. All cases were assessed according to the Union for International Cancer Control/American Joint Committee on Cancer TNM Classification ( 7 th ed). ${ }^{7}$ Resected specimens were formalin-fixed and stained with hematoxylin and eosin in a routine manner. Histologic examinations were performed according to the newly proposed IASLC/ATS/ERS classification of lung adenocarcinoma. ${ }^{5}$ Specimens were evaluated pathologically by a single pathologist (M.O.). The sizes of both the total tumor and the invasive component were verified for each tumor by 2 doctors (M.O. and M.T.). In this study, adenocarcinoma in situ (AIS) and minimally invasive adenocarcinoma (MIA) were referred to as noninvasive adenocarcinoma, and the others types of tumors were referred to as invasive adenocarcinoma.

Routine preoperative tests performed were as follows: chest roentgenography, laboratory tests including those for serum carcinoembryonic antigen (CEA) level, enhanced CT of the whole body, and enhanced brain magnetic resonance imaging. Either positron emission tomography with F-18 fluorodeoxyglucose or bone scintigraphy was performed for all patients. Preoperative serum CEA levels were assessed using enzymelinked immunosorbent assay kits, with an upper limit of normal of $5.0 \mathrm{ng} / \mathrm{mL}$.

Contrast-enhanced chest $\mathrm{CT}$ images were obtained using a commercially available scanner (Brilliance $64 \mathrm{CT}$ scanner; Philips Electronics Japan, Tokyo, Japan). Thin-section CT images targeted to the tumor were obtained for each patient using the following parameters: $120 \mathrm{kVp}, 490 \mathrm{~mA}, 1-\mathrm{mm}$ section thickness, and $512 \times 512$ pixel resolution. The images were assessed using the mediastinal window setting (level, 40 Hounsfield units [HU]; width, $350 \mathrm{HU}$ ) and the lung window setting (level, $-600 \mathrm{HU}$; width, $1500 \mathrm{HU}$ ). Ground-glass opacity (GGO) was defined as a hazy increase in attenuation not associated with obscured underlying lung structures in the lung window setting, whereas consolidation was defined as an increase in attenuation that obscured the underlying structures. ${ }^{8}$

In the present study, we examined 3 radiologic parameters: the GGO ratio, tumor disappearance rate (TDR), and consolidation diameter (CD). The GGO ratio $(\%)$ was defined as follows: $(1-$ [maximum dimension of consolidation on the lung window setting/maximum dimension of the tumor on the lung window setting]) $\times 100$. $^{9}$ The TDR $(\%)$ was defined as follows: $(1-$ [maximum area of consolidation on the mediastinal window setting/maximum area of tumor on the lung window setting] $\times 100 .^{9,10}$ The $\mathrm{CD}(\mathrm{mm})$ was defined as the largest diameter of consolidation on the lung window setting. Preoperative CT images were reviewed by 2 doctors (M.T. and Y.S.) blinded to the histopathologic status. Wide interobserver discrepancies in calculating the radiologic parameters were resolved by consensus with a third observer. Patients were followed up on an outpatient basis at least every 6 months to evaluate recurrence and survival. Chest CT was performed at 6-month intervals. Patients with recurrent disease received chemotherapy, radiotherapy, or both as often as possible.

The survival probability was calculated using the Kaplan-Meier method and differences in survival were evaluated using the log-rank test. Survival was calculated from the date of surgical intervention until death from any cause. To predict invasive adenocarcinomas defined according to the new IASLC/ATS/ERS classification, receiver operating characteristic (ROC) curves for the GGO ratio, TDR, and CD were generated. The optimal cut-off points were defined as those on the curves closest to the upper left-hand corner. ${ }^{11}$ Statistical measures and significance based on these cut-off values were investigated using binary classification and the chi-square test. We performed univariate logistic regression analysis to examine the predictive value of radiologic parameters and conventional clinical factors for pathologically invasive adenocarcinomas. Statistical analysis was performed using SPSS version 19 (Chicago, Ill). A $P$ value of less than .05 was considered statistically significant.

\section{RESULTS}

The clinical and pathologic characteristics of the patients are summarized in Table 1 . We detected 54 noninvasive adenocarcinomas and 69 invasive adenocarcinomas. The 5 -year overall survival rate for all patients was $88.5 \%$ (95\% confidence interval [CI], 80.8\%-96.2\%). Tumor recurrences were found in 8 patients $(6.5 \%)$, and in 5 cases recurrence resulted in cancer-related deaths. No recurrence was detected in patients with noninvasive adenocarcinomas (lobectomy, 30; limited resection, 24). Among the patients with noninvasive adenocarcinomas, the 5-year overall survival rate was $100 \%$. In contrast, the 5 -year overall survival rate was $78.4 \%$ (95\% CI, $64.5 \%-92.2 \%)$ for patients with invasive adenocarcinomas (lobectomy, 53; limited resection, 16). This difference reached statistical significance $(P<.01$; Figure 2$)$, indicating possible prognostic relevance.

The ROC curves in Figure 3 show the true-positive ratio (sensitivity), and the false-positive ratio ( 1 - specificity) for the GGO ratio, TDR, and CD to predict pathologically invasive adenocarcinomas. The optimal cut-off values for the GGO ratio, TDR, and CD according to the ROC curves were $50 \%$ (area under the curve [AUC], $0.91 ; 95 \% \mathrm{CI}$, 0.86-0.97), 75\% (AUC, 0.90; 95\% CI, 0.84-0.96), and 10 mm (AUC, 0.91; 95\% CI, 0.86-0.97), respectively. As the AUC for each parameter approached 0.9, the cut-off values were considered highly accurate. The relationship between the radiologic parameters and pathologic invasiveness of lung adenocarcinoma according to the cut-off values was investigated (Table 2). With these cut-off values, the GGO ratio, TDR, and $\mathrm{CD}$ showed positive predictive values greater than $90 \%$. The results of univariate analysis to predict invasive adenocarcinoma using these cut-off values 


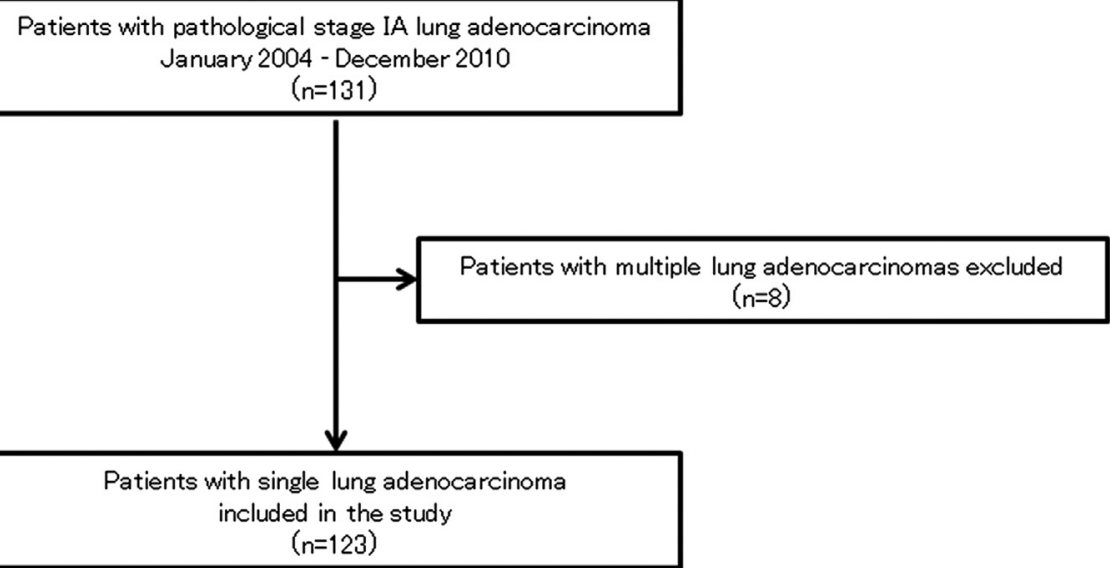

FIGURE 1. Patient selection flow chart.

and conventional clinical factors is shown in Table 3. Clinically significant predictors of invasive adenocarcinoma were the GGO ratio $(\leq 50 \%$ vs $>50 \% ; P<.01)$, TDR $(\leq 75 \%$ vs $>75 \% ; P<.01), \mathrm{CD}(\leq 10 \mathrm{~mm}$ vs $>10 \mathrm{~mm}$; $P<.01)$, tumor size $(\leq 20 \mathrm{~mm}$ vs $>20 \mathrm{~mm} ; P=.048)$, and preoperative serum CEA level $(\leq 5 \mathrm{ng} / \mathrm{mL}$ vs $>5 \mathrm{ng} / \mathrm{mL} ; P<.01)$.

TABLE 1. Clinicopathologic characteristics of the patients

\begin{tabular}{|c|c|c|c|}
\hline Factor & $\begin{array}{c}\text { Noninvasive } \\
(\mathbf{N}=\mathbf{5 4})\end{array}$ & $\begin{array}{l}\text { Invasive } \\
(\mathbf{N}=69)\end{array}$ & $\begin{array}{c}\text { Total } \\
(\mathbf{N}=\mathbf{1 2 3}) \\
\end{array}$ \\
\hline \multicolumn{4}{|l|}{ Sex } \\
\hline Male & 15 & 41 & 56 \\
\hline Female & 39 & 28 & 67 \\
\hline \multicolumn{4}{|l|}{ Age (y) } \\
\hline$\leq 70$ & 39 & 36 & 75 \\
\hline$>70$ & 15 & 33 & 48 \\
\hline \multicolumn{4}{|l|}{ Surgical procedure } \\
\hline Lobectomy & 30 & 53 & 83 \\
\hline Limited resection & 24 & 16 & 40 \\
\hline \multicolumn{4}{|l|}{ Tumor diameter (mm) } \\
\hline$\leq 20$ & 43 & 41 & 84 \\
\hline$>20$ & 11 & 28 & 39 \\
\hline \multicolumn{4}{|l|}{ Preoperative serum CEA level } \\
\hline Normal & 51 & 50 & 101 \\
\hline Increased & 3 & 19 & 22 \\
\hline Recurrence & 0 & 8 & 8 \\
\hline 5 -y overall survival rate $(\%)$ & 100 & 78.4 & 88.5 \\
\hline \multicolumn{4}{|l|}{ Histologic type } \\
\hline Adenocarcinoma in situ & 27 & - & \\
\hline Minimally invasive adenocarcinoma & 27 & - & \\
\hline Lepidic predominant & - & 10 & \\
\hline Acinar predominant & - & 14 & \\
\hline Papillary predominant & - & 30 & \\
\hline Micropapillary predominant & - & 2 & \\
\hline Solid predominant & - & 4 & \\
\hline Invasive mucinous adenocarcinoma & - & 9 & \\
\hline
\end{tabular}

Limited resection included wedge resection and segmentectomy. $N$, Number of patients; $C E A$, carcinoembryonic antigen.

\section{DISCUSSION}

Our results suggested 2 outcomes. First, tumor invasiveness as defined according to the IASLC/ATS/ERS classification of adenocarcinoma was identified as a significant prognostic factor for p-stage IA disease. Second, the GGO ratio, TDR, and CD on thin-section CT were well correlated with tumor invasiveness and could be useful for detecting invasive adenocarcinoma.

In the new IASLC/ATS/ERS classification, one major transition is the discontinuation of the term "bronchioloalveolar carcinoma" (BAC). One of the reasons for this is that the term BAC has been applied to a broad spectrum of tumors, ranging from solitary small noninvasive lung adenocarcinomas to mixed subtype invasive adenocarcinomas. ${ }^{3,5}$

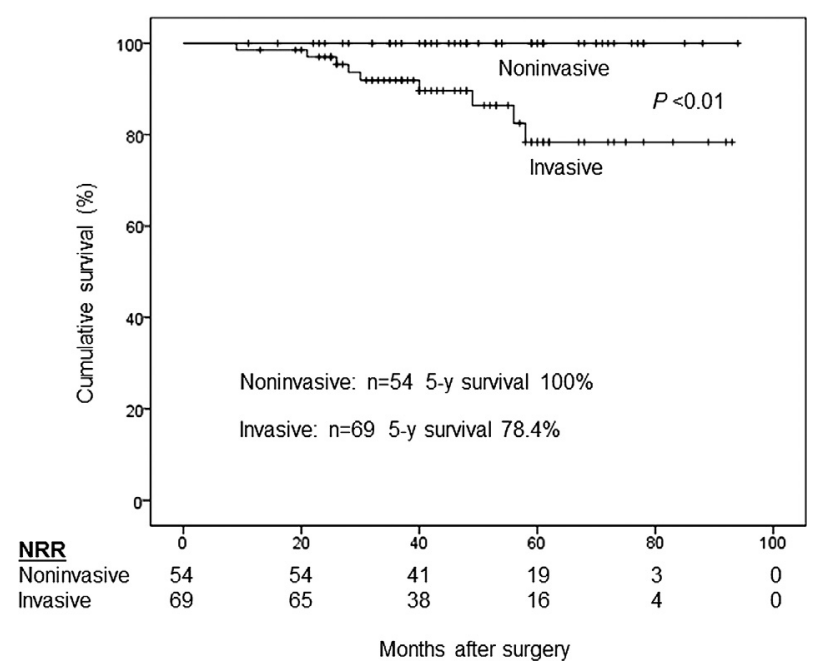

FIGURE 2. Overall survival curves of patients with p-stage IA adenocarcinoma based on tumor invasiveness proposed by the International Association for the Study of Lung Cancer, American Thoracic Society and European Respiratory Society. A significant difference was noted between noninvasive adenocarcinoma and invasive adenocarcinoma $(P<.01)$. NRR, Number remaining at risk. 

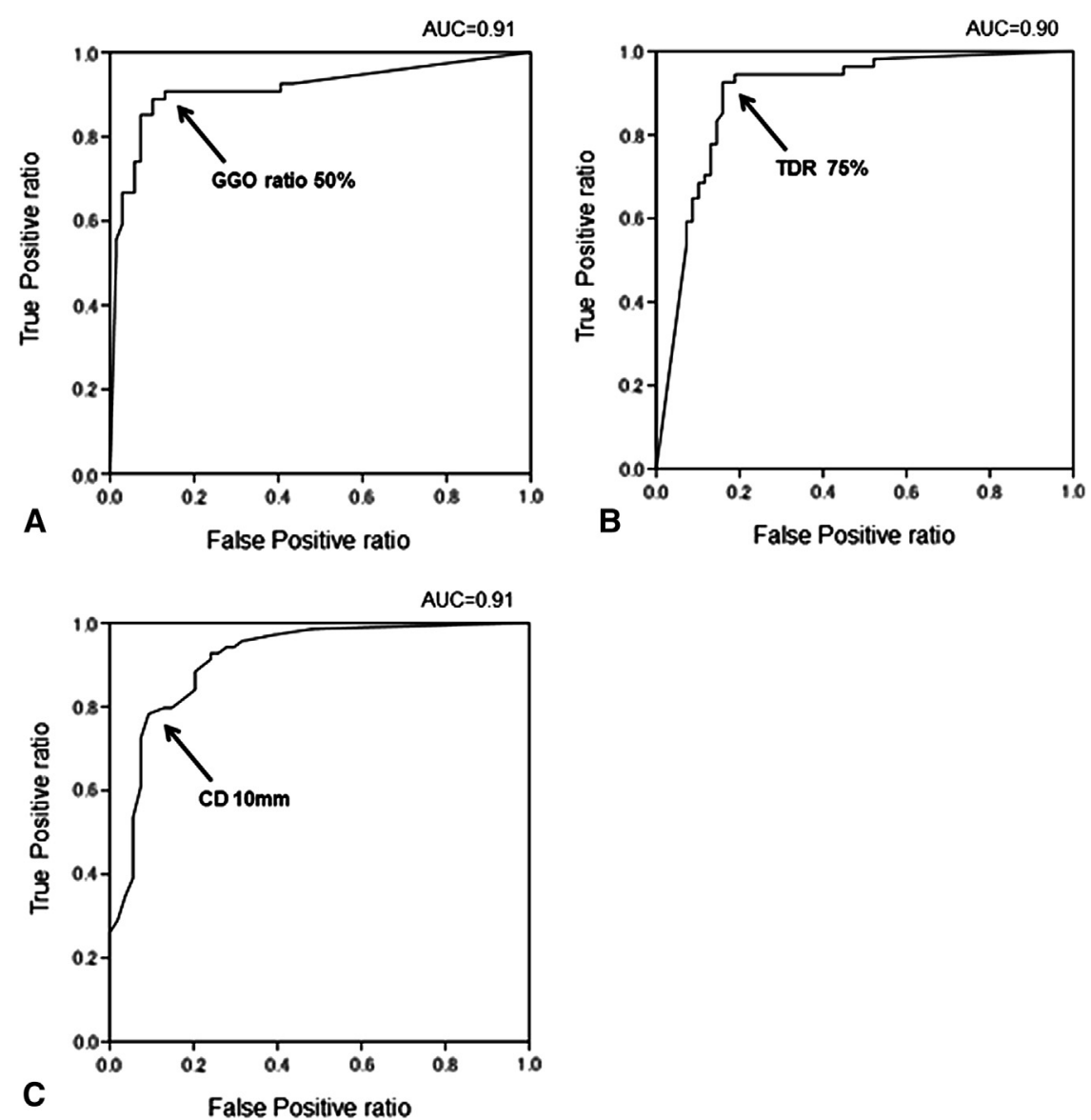

FIGURE 3. Receiver operating characteristic curves for the (A) GGO ratio, (B) TDR, and (C) CD showing the optimal cut-off values for the parameters. $A U C$, Area under the curve; $G G O$, ground-glass opacity; $T D R$, tumor disappearance rate; $C D$, consolidation diameter.

The new IASLC/ATS/ERS classification has proposed AIS and MIA instead of BAC to define noninvasive adenocarcinomas.

Our study confirmed a clear prognostic difference between noninvasive adenocarcinoma and invasive adenocarcinoma as defined according to the new IASLC/ATS/ ERS classification. This finding supported the classification system with regard to the identification of histologic subtypes of lung adenocarcinoma with prognostic value.

In addition, no recurrences were found in noninvasive adenocarcinomas regardless of the surgical procedures used in the present study. AIS and MIA were suggested to be possible candidates for limited resection because the prognoses were excellent with complete resection in our study and in previous reports. ${ }^{4,6}$ In fact, lung-sparing resections have been indispensable procedures for patients with multiple noninvasive adenocarcinomas. ${ }^{12-14}$ However, the standard surgical procedure for lung cancer should be lobectomy and only carefully selected patients should undergo limited resection. Therefore, preoperative or intraoperative evaluation can be crucial in selecting surgical procedures.
Generally, it is very difficult for pathologists to exclude tumor invasion on the basis of an examination of a preoperative or intraoperative frozen section. ${ }^{6}$ Thus, the radiologic features of noninvasive and invasive adenocarcinomas on preoperative radiography may help determine the most appropriate surgical procedures.

The second finding of the present study was that thin-section CT features were well correlated with tumor invasiveness as defined by the new IASLC/ATS/ERS classification. Difficulty in preoperatively identifying invasive tumors according to radiologic findings has been highlighted in several studies. ${ }^{15,16}$ This may be because tumor size alone is not an accurate indicator of tumor invasiveness. ${ }^{15}$

Recently, improved understanding of the pathologic and radiologic characteristics of small adenocarcinomas of the lung has clarified the correlation between thin-section CT findings and pathologic features. ${ }^{17-20}$ The lepidic growth pattern of adenocarcinoma replacing the alveolar wall presents a GGO appearance on thin-section CT. In contrast, a solid tumor appearance on thin-section CT reflects a collapse of the alveolar wall, fibrosis, and proliferation of 
TABLE 2. Relationship between radiologic parameters and pathologic invasiveness of lung adenocarcinoma

\begin{tabular}{|c|c|c|c|c|c|c|}
\hline Radiologic parameter & Noninvasive $(\mathbf{N})$ & Invasive $(\mathbf{N})$ & Sensitivity $(\%)$ & Specificity $(\%)$ & PPV $(\%)$ & FNR $(\%)$ \\
\hline \multicolumn{7}{|l|}{ Ground-glass opacity ratio } \\
\hline$>50 \%$ & 48 & 8 & 88.4 & 88.9 & 92.4 & 11.6 \\
\hline$\leq 50 \%$ & 6 & 61 & & & & \\
\hline \multicolumn{7}{|l|}{ Tumor disappearance rate } \\
\hline$>75 \%$ & 51 & 14 & 79.7 & 94.4 & 94.8 & 20.3 \\
\hline$\leq 75 \%$ & 3 & 55 & & & & \\
\hline \multicolumn{7}{|l|}{ Consolidation diameter } \\
\hline$>10 \mathrm{~mm}$ & 5 & 54 & 78.3 & 90.7 & 91.5 & 21.7 \\
\hline$\leq 10 \mathrm{~mm}$ & 49 & 15 & & & & \\
\hline
\end{tabular}

$N$, Number of patients; $P P V$, positive predictive value; $F N R$, false-negative rate

invasive tumor cells. ${ }^{19}$ The extent of central fibrosis is associated significantly with surgical outcomes. ${ }^{21,22}$ Persuasive data for evaluating the percentage of the GGO component on preoperative radiography would be more meaningful than ever before because the IASLC/ATS/ ERS have proposed new clear definitions for invasive adenocarcinoma based on intensive analysis of a large database. Thus, it is likely that this pathologic classification will affect treatment strategy. ${ }^{6}$

To date, few reports have examined the relationship between radiologic features and pathologic status based on the new classification. ${ }^{2}$ Our data indicated that the GGO ratio, TDR, and $\mathrm{CD}$ were strong indicators of tumor invasiveness according to the IASLC/ATS/ERS classification and that a GGO ratio of $50 \%$, a TDR of $75 \%$, and a $\mathrm{CD}$ of $10 \mathrm{~mm}$ could be practical cut-off points. In fact, previous studies have shown that a GGO ratio or a TDR greater than $50 \%$ for small adenocarcinomas was a favorable prognostic factor. ${ }^{17-20}$ Our data, based on the new pathologic classification, support these results.

Although our findings indicated that all 3 parameters on thin-section CT were fully correlated with tumor invasiveness, several reports have suggested that the TDR more strongly correlated with pathologic invasiveness than the GGO ratio. ${ }^{17,18}$ In our analysis, the GGO ratio, TDR, and $\mathrm{CD}$ all were associated because tumors with a high GGO ratio generally showed high TDR and low CD. Thus, we recognized that comparing these parameters by multivariate analysis would not be statistically meaningful for predicting invasive adenocarcinoma in this situation. Our results suggested that radiologic parameters based on
GGO might be useful regardless of dimensionality, although many radiologic parameters, such as the single largest diameter, bidimensionality, and volume, have been tested. $^{2,5}$

Our study had several limitations. First, this study was retrospective and selection bias should not be ignored. For example, a greater fraction of patients with noninvasive adenocarcinoma had limited resection compared with those with invasive cancer. This suggests that we offered limited resection to patients with favorable radiographic findings. Thus, it will not be suitable to evaluate surgical indications according to the results of the current study. Surgical indicators for limited resection should be strictly verified in randomized prospective studies. At present, phase III randomized trials of standard lobectomy versus experimental limited resection for small lung cancer are ongoing in the United States (CALGB-140503) and in Japan (JCOG 0804/WJOG4607L). Retrospective cumulative data, including data from the present study, may be warranted before the outcomes of these prospective studies are confirmed in years to come. Second, the sample size was relatively small. Nevertheless, our overall 5-year survival data of $88.5 \%$ for p-stage IA lung adenocarcinoma were equivalent to those of previous reports based on a larger database. ${ }^{23,24}$ In the current study, all surgeries were performed or supervised by the same surgeon and all specimens were evaluated pathologically by the same pathologist to ensure quality of the treatment and the pathologic examination. Third, interobserver variability when calculating the parameters cannot be ignored. The border of the GGO shadow can be so obscure that estimating tumor size is

TABLE 3. Univariate analysis of possible predictors for invasive adenocarcinoma

\begin{tabular}{lcccc}
\hline \multicolumn{1}{c}{ Factor } & Favorable & Unfavorable & Odds ratio $(\mathbf{9 5} \%$ CI) & $\boldsymbol{P}$ value \\
\hline Tumor size & $\leq 20 \mathrm{~mm}$ & $>20 \mathrm{~mm}$ & $2.3(1.1-5.0)$ & .048 \\
Ground-glass opacity ratio & $>50 \%$ & $\leq 50 \%$ & $65.3(20.7-207.7)$ & $<.01$ \\
Tumor disappearance rate & $>75 \%$ & $\leq 75 \%$ & $30.4(10.3-89.7)$ & $26.4(9.8-70.8)$ \\
Consolidation diameter & $\leq 10 \mathrm{~mm}$ & $>10 \mathrm{~mm}$ & $6.5(1.8-23.2)$ & $<.01$ \\
Preoperative serum CEA level & $\leq 5 \mathrm{ng} / \mathrm{mL}$ & $>5 \mathrm{ng} / \mathrm{mL}$ & $<.01$ \\
\hline
\end{tabular}

$C I$, Confidence interval; $C E A$, carcinoembryonic antigen. 
subject to considerable variability. We retained the defined window settings on the $\mathrm{CT}$ image for each observer to minimize the possibility of measurement errors. For many of the parameters, we applied the GGO ratio, TDR, and $\mathrm{CD}$ to emphasize reproducibility and simplicity.

In conclusion, tumor invasiveness as defined according to the IASLC/ATS/ERS classification of adenocarcinoma was a significant prognostic factor for $\mathrm{p}$-stage IA adenocarcinoma. The GGO ratio, TDR, and CD can be useful predictors of invasive adenocarcinoma.

The authors would like to thank Dr Toshihiko Sato, Department of Thoracic Surgery, Graduate School of Medicine, Kyoto University, for his manuscript review.

\section{References}

1. Noguchi M, Morikawa A, Kawasaki M, Matsuno Y, Yamada T, Hirohashi S, et al. Small adenocarcinoma of the lung. Histologic characteristics and prognosis. Cancer. 1995; 75:2844-52

2. Honda T, Kondo T, Murakami S, Saito H, Oshita F, Ito H, et al. Radiographic and pathological analysis of small lung adenocarcinoma using the new IASLC classification. Clin Radiol. 2013;68:e21-6.

3. Travis WD, Brambilla E, Müller-Hermelink HK, Harris CC, eds. World Health Organization Classification of Tumours. Pathology and genetics: tumours of the lung, pleura, thymus and heart. Lyon: IARC; 2004.

4. Wu J, Ohta Y, Minato H, Tsunezuka Y, Oda M, Watanabe Y, et al. Nodal occult metastasis in patients with peripheral lung adenocarcinoma of $2.0 \mathrm{~cm}$ or less in diameter. Ann Thorac Surg. 2001;71:1772-8.

5. Travis WD, Brambilla E, Noguchi M, Nicholson AG, Geisinger KR, Yatabe Y, et al. International Association for the Study of Lung Cancer/American Thoracic Society/European Respiratory Society international multidisciplinary classification of lung adenocarcinoma. J Thorac Oncol. 2011;6:244-85.

6. Yoshizawa A, Motoi N, Riely GJ, Sima CS, Gerald WL, Kris MG, et al. Impact of proposed IASLC/ATS/ERS classification of lung adenocarcinoma: prognostic subgroups and implications for further revision of staging based on analysis of 514 stage I cases. Mod Pathol. 2011;24:653-64.

7. Sobin L, Gospodarowicz M, Wittekind C. International Union Against Cancer (UICC): TNM classification of malignant tumours. 7th ed. New York: Wiley; 2002.

8. Kuriyama K, Seto M, Kasugai T, Higashiyama M, Kido S, Sawai Y, et al. Ground-glass opacity on thin section CT: value in differentiating subtypes of adenocarcinoma of the lung. AJR Am J Roentgenol. 1999;173:465-9.

9. Okada M, Nakayama H, Okumura S, Daisaki H, Adachi S, Yoshimura M, et al. Multicenter analysis of high-resolution computed tomography and positron emission tomography/computed tomography findings to choose therapeutic strategies for clinical stage IA lung adenocarcinoma. J Thorac Cardiovasc Surg. 2011;141:1384-91.
10. Takamochi K, Nagai K, Yoshida J, Suzuki K, Ohde Y, Nishimura M, et al Pathologic N0 status in pulmonary adenocarcinoma is predictable by combining serum carcinoembryonic antigen level and computed tomographic findings. J Thorac Cardiovasc Surg. 2001;122:325-30.

11. Akobeng AK. Understanding diagnostic tests 3: receiver operating characteristic curves. Acta Paediatr. 2007;96:644-7.

12. Mun M, Kohn T. Efficacy of thoracoscopic resection for multifocal bronchioloalveolar carcinoma showing pure ground-glass opacities of $20 \mathrm{~mm}$ or less in diameter. J Thorac Cardiovasc Surg. 2007;134:877-82.

13. Okada M, Koike T, Higashiyama M, Yamato Y, Kodama K, Tsubota N. Radical sublobar resection for small-sized non-small cell lung cancer: a multicenter study. J Thorac Cardiovasc Surg. 2006;132:769-75.

14. Sienel W, Dango S, Kirschbaum A, Cucuruz B, Hörth W, Stremmel C, et al Sublobar resections in stage IA non-small cell lung cancer: segmentectomies result in significantly better cancer-related survival than wedge resections. Eur J Cardiothorac Surg. 2008;33:728-34.

15. Yoshida J, Nagai K, Yokose T, Nishimura M, Kakinuma R, Ohmatsu H, et al Limited resection trial for pulmonary ground-glass opacity nodules: fifty-case experience. J Thorac Cardiovasc Surg. 2005;129:991-6.

16. Nakata M, Sawada S, Saeki H, Takashima S, Mogami H, Teramoto N, et al Prospective study of thoracoscopic limited resection for ground-glass opacity selected by computed tomography. Ann Thorac Surg. 2003;75 1601-6.

17. Okada M, Nishio W, Sakamoto T, Uchino K, Hanioka K, Ohbayashi C, et al Correlation between computed tomographic findings, bronchioloalveolar carcinoma component, and biologic behavior of small-sized lung adenocarcinomas. J Thorac Cardiovasc Surg. 2004;127:857-61

18. Nakayama H, Yamada K, Saito H, Oshita F, Ito H, Kameda Y, et al. Sublobar resection for patients with peripheral small adenocarcinomas of the lung: surgical outcome is associated with features on computed tomographic imaging. Ann Thorac Surg. 2007;84:1675-9.

19. Hashizume T, Yamada K, Okamoto N, Saito H, Oshita F, Kato Y, et al. Prognostic significance of thin-section CT scan findings in small-sized lung adenocarcinoma. Chest. 2008;133:441-7.

20. Matsuguma H, Yokoi K, Anraku M, Kondo T, Kamiyama Y, Mori K, et al. Proportion of ground-glass opacity on high-resolution computed tomography in clinical T1N0M0 adenocarcinoma of the lung: a predictor of lymph node metastasis. J Thorac Cardiovasc Surg. 2002;124:278-84.

21. Suzuki K, Yokose T, Yoshida J, Nishimura M, Takahashi K, Nagai K, et al Prognostic significance of the size of central fibrosis in peripheral adenocarcinoma of the lung. Ann Thorac Surg. 2000;69:893-7.

22. Borczuk A, Qian F, Kazeros A, Eleazar J, Assaad A, Sonett JR, et al. Invasive size is an independent predictor of survival in pulmonary adenocarcinoma. Am J Surg Pathol. 2009;33:462-9

23. Goldstraw P, Crowley J, Chansky K, Giroux DJ, Groome PA, Rami-Porta R, et al The IASLC lung cancer staging project: proposals for the revision of the TNM stage groupings in the forthcoming (seventh) edition of the TNM classification of malignant tumours. J Thorac Oncol. 2007;2:706-14.

24. Kameyama K, Takahashi M, Ohata K, Igai H, Yamashina A, Matsuoka T, et al. Evaluation of the new TNM staging system proposed by the international association for the study of lung cancer at a single institution. J Thorac Cardiovasc Surg. 2009;137:1180-4. 